\title{
Optimization of Fluorescent Proteins and Techniques for In-resin Correlative Light and Electron Microscopy
}

\author{
Maria Paez-Segala ${ }^{1}$, Yalin Wang ${ }^{2}$, Nirmala Iyer ${ }^{1}$, Wei-Ping Li $^{1}$, Patricia Rivlin ${ }^{1}$ and Loren Looger ${ }^{1}$ \\ ${ }^{1}$ Janelia Research Campus-HHMI, Ashburn, Virginia, United States, ${ }^{2}$ Tsinghua University, Haidian \\ District, Beijing, China (People's Republic)
}

Correlative light and electron microscopy (CLEM) combines the power of electron microscopy, with its excellent resolution and contrast, with that of fluorescence imaging, which allows the staining of specific molecules, organelles, and cell populations. Fluorescence imaging is also readily compatible with live cells and behaving animals, facilitating real-time visualization of cellular processes, potentially followed by electron microscopic reconstruction. Super-resolution single-molecule localization microscopy is a relatively new modality that harnesses the ability of some fluorophores to photoconvert, through which localization precision better than Abbe's diffraction limit is achieved through iterative high-resolution localization of single-molecule emitters. Here we describe our lab's recent progress in the development of reagents and techniques for super-resolution single-molecule localization CLEM and their applications to biological problems.

We have previously engineered mutants of the Eos green-to-red photoconvertible fluorescent protein (mEos4a and mEos4b) [1] that preserve a large fraction of their fluorescence and photoconverting behavior following strenuous preparation for EM, including aldehyde fixation (4\% paraformaldehyde, $0.2 \%$ glutaraldehyde), post-fixation with $1 \% \mathrm{OsO}_{4}$ to preserve and enhance membrane contrast, en blocstaining with $1 \%$ uranyl acetate, and embedding in plastic resin (glycol methacrylate, GMA) for ultrastructure preservation, long-term storage stability, and sectioning.

These fluorescent labels and techniques permit a number of experiments [2], but there is much room for improvement: i) we had only demonstrated the method on cultured cells, not on intact tissue; ii) the fixation resistance of the probes should be improved; and iii) GMA and other acrylate resins were initially used because they tolerate a little water during polymerization (which can be important for proper function of fluorescent proteins and antibodies), but produce fairly soft plastics, leading to sub-optimal ultrastructure preservation and difficulty cutting numerous sections from a single block (100's to 1000's are often required for reconstruction of neuronal arbors across a local circuit, for instance) - epoxy resins such as Epon are the gold standard for EM reconstruction. Here we describe the extension of our method first to Lowicryl HM20 resin (with better ultrastructure preservation than GMA), then to the analysis of the intact neuromuscular junction (NMJ) of Drosophilalarvae in HM20 resin, and finally to the engineering of "Eos5" variants with much greater fixation resistance, surviving well even in Epon resin.

The Drosophilalarval NMJ is a classic preparation for ultrastructural examination; for instance, the individual muscles and their corresponding motor neurons are well-studied. A number of unanswered questions remain, however; for example, the complement of synaptic vesicles in the axonal boutons show characteristic, reproducible differences in appearance across motor neurons and individual boutons $[3,4]$, but the precise molecular composition of these synaptic vesicles is not understood, nor are the specifics of their association with endogenous machinery. We chose this preparation as a testbed for super- 
resolution CLEM techniques, both as a rigorous method to characterize new reagents in a system with a good amount of known ground truth, and also to shed light on unknown biology.

Figure 1 shows CLEM of mitochondrially expressed mEos4a in the axonal boutons of a single motor

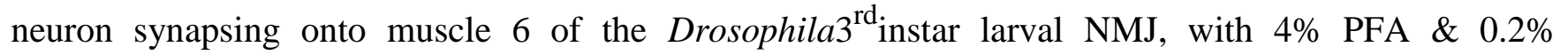
glutaraldehyde fix, $0.5 \% \mathrm{OsO}_{4} \& 0.1 \%$ UAc post-fix, complete dehydration, and embedding in Lowicryl HM20 resin. Fluorescence corresponds exactly with boutons in EM (Fig. 1c).

Figure 2 shows performance of an unpublished, improved Eos variant ("Eos5"), in HeLa cells in HM20 and Epon 812 resins. The new "Eos5" variant is much more fixation-resistant than the mEos 4 variants (Fig. 2f,g), and retains its green-to-red photoconvertibility in Epon resin (Fig. 2h). "Eos5" supports CLEM with the excellent ultrastructure preservation provided by Epon and other epoxy resins (Fig. 2k).
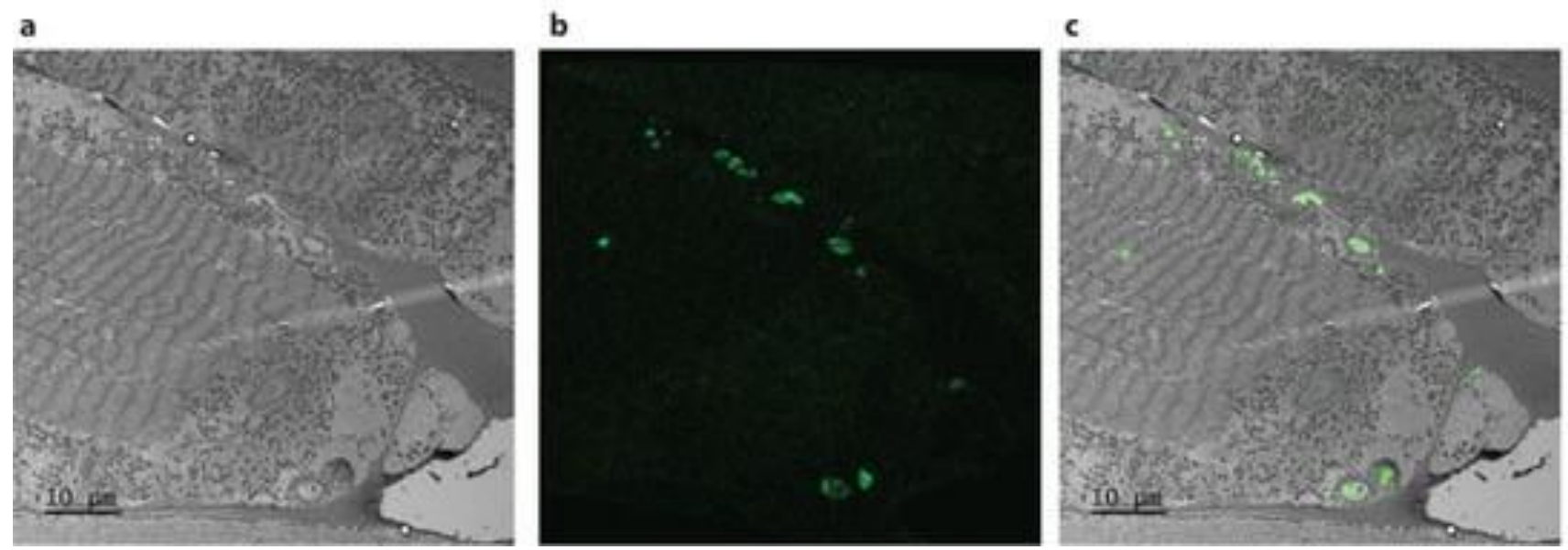

Figure 1. CLEM of bouton string in the fly neuromuscular junction. CLEM on a 3rd instar Drosophila larva expressing (pan-neuronally) the mEos4a fluorescent protein in a motor neuron making a string of axonal boutons onto muscles 6 (lower left) \& 7 (upper right). Larva prepared by i) fixation with $4 \%$ PFA \& $0.2 \%$ glutaraldehyde, ii) high-pressure freezing, iii) freeze-substitution with $0.5 \%$ OsO4\& $0.1 \%$ UAc, iv) complete dehydration with $100 \%$ acetone, v) infiltration and UV polymerization with Lowicryl HM20 resin, and vi) cutting of sections. a) Confocal fluorescence image of a $100 \mathrm{~nm}$ section. b) TEM micrograph image of the next section in the block (50 nm thick). c) Composite image assembled using ecCLEM software (Heiligenstein et. al, 2017). Note the diverse structures of the labeled axonal boutons along muscle 6, all arising from a single motor neuron. Also clearly visible are muscle striations and mitochondria. 

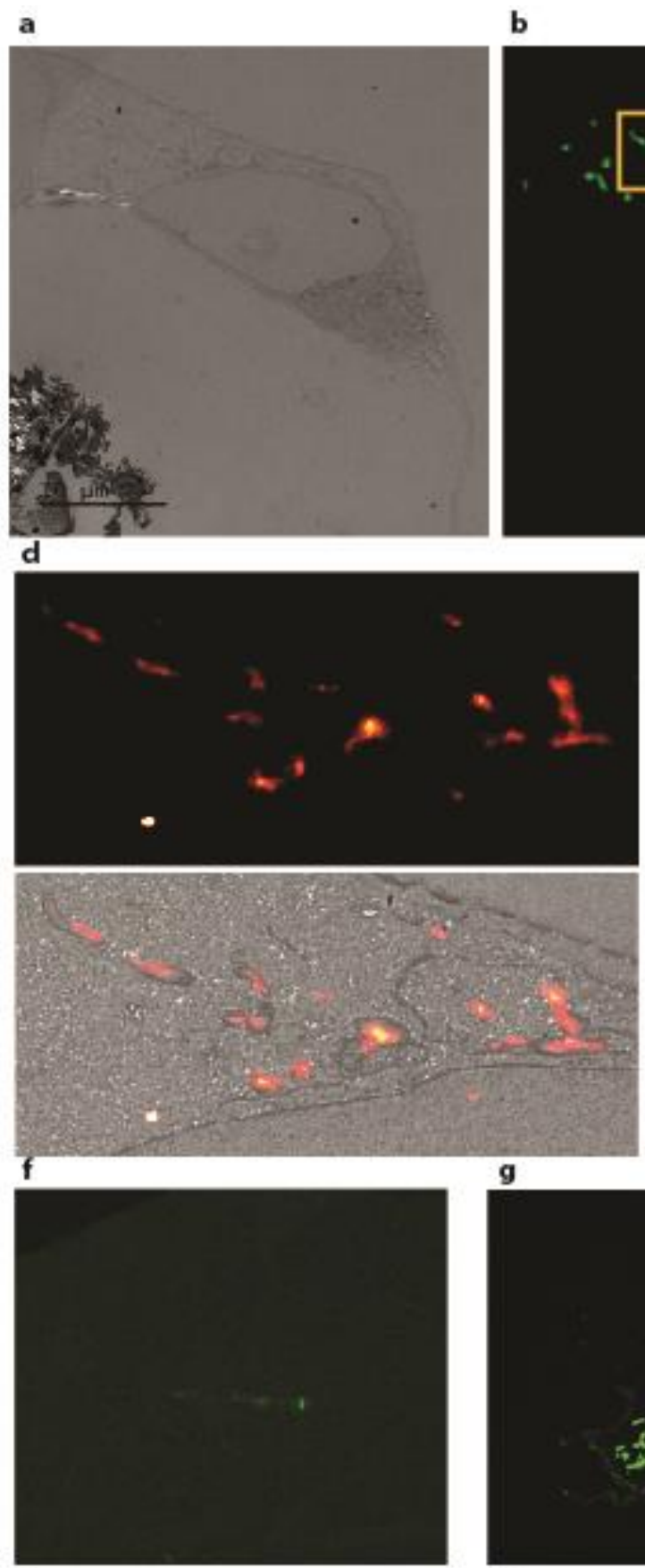

i

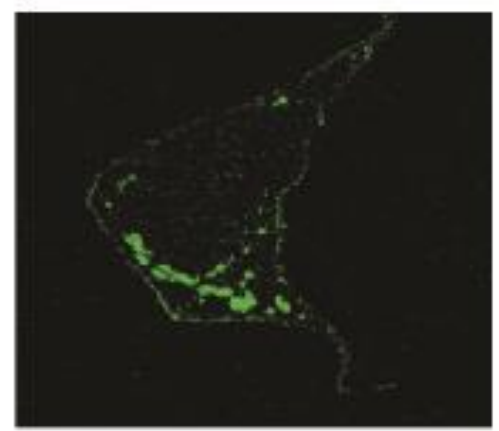

b

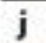

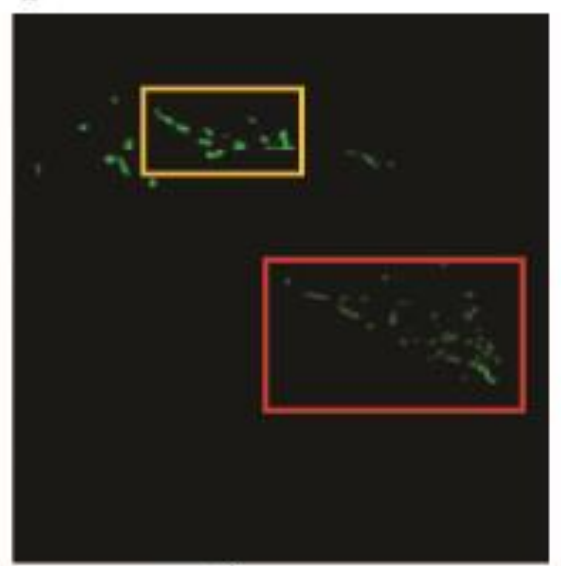

e
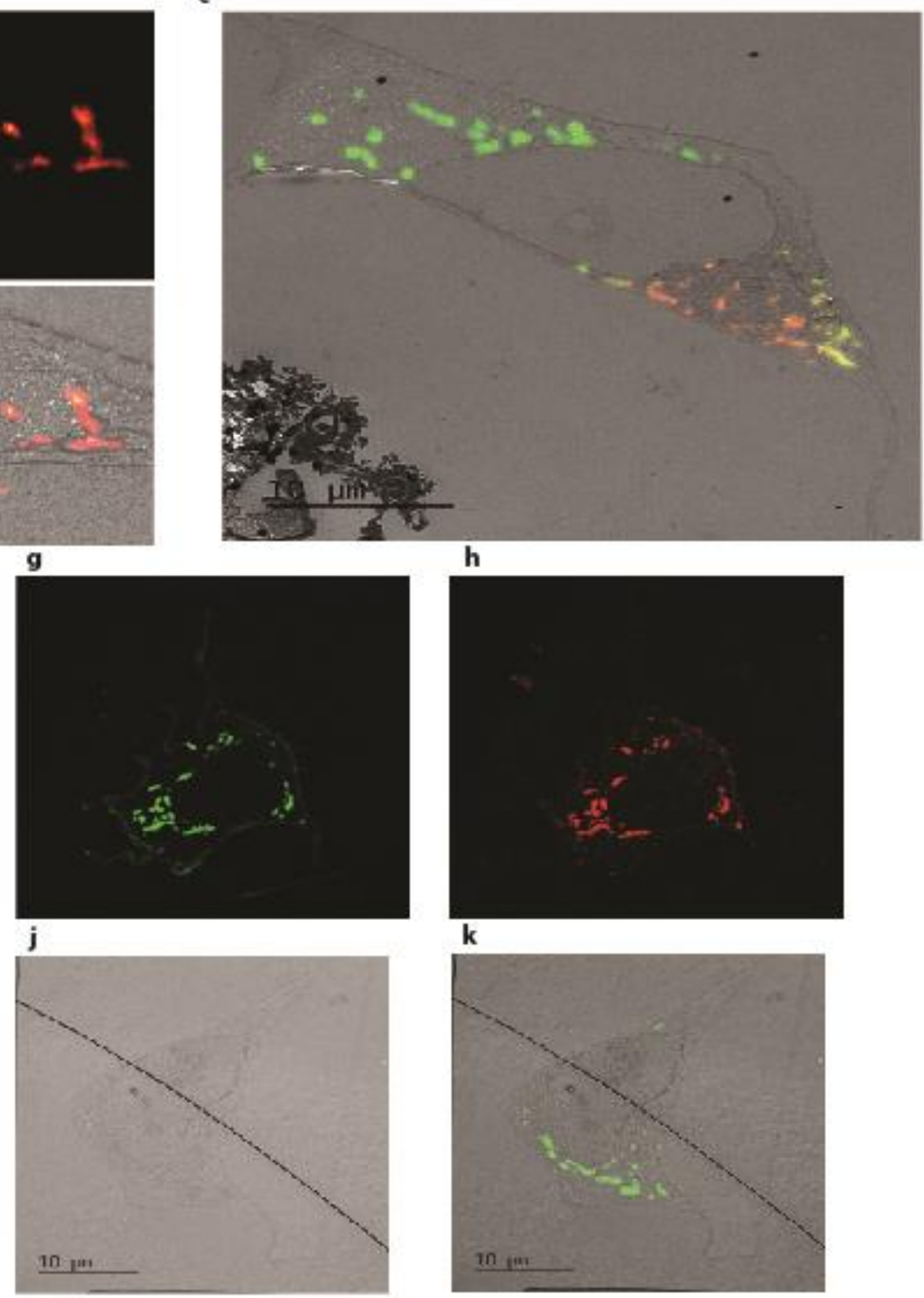

h

c
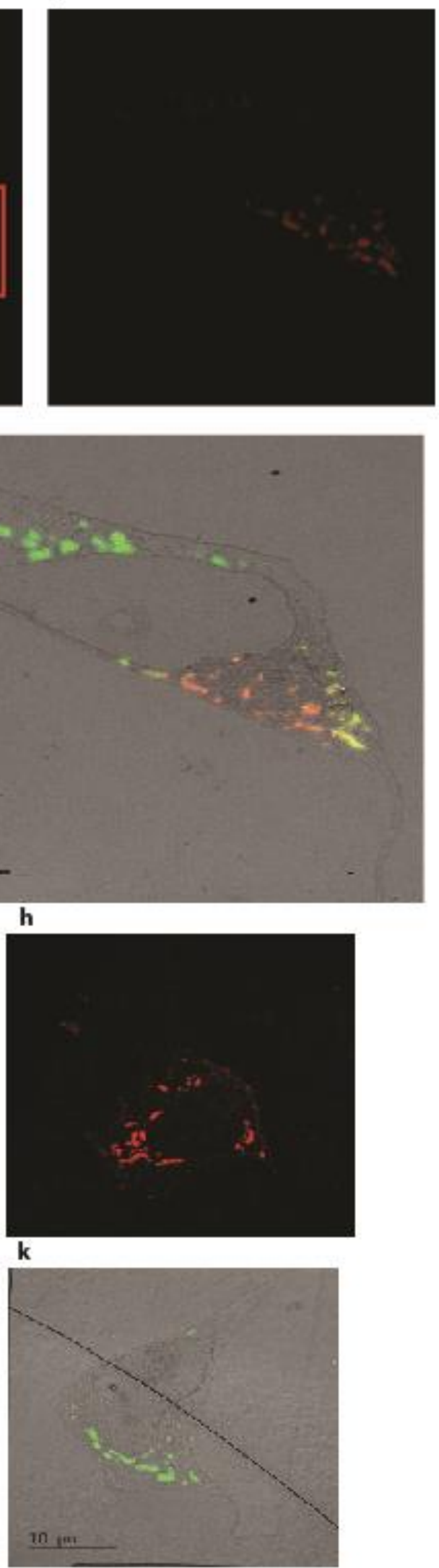
Figure 2. Performance of new "Eos5" variant in CLEM in HM20 and Epon resins. Panels a-e: a single HeLa cell with mitochondrially expressed "mEos5", in HM20 resin as in Fig. 1. a) TEM image. b) Confocal fluorescence image of the same field of view in the green channel. Orange box at top shows region with super-resolution PALM image shown in panel d. Bottom red box shows region UVphotoconverted to the red state shown in panel c. c) Confocal fluorescence image of the same field of view in the red channel after photoconversion. d-top) Super-resolution PALM image of the orange-boxed region from panel b. d-bottom) CLEM of PALM imaging. e) CLEM of confocal imaging in both the green and red channels. Panels f-h: comparison of mEos4a and "Eos5" in Epon 812 resin, following similar preservation with $4 \%$ PFA \& $0.2 \%$ glutaraldehyde, high-pressure freezing, $0.5 \%$ OsO4\& $0.1 \%$ UAc, complete dehydration with $100 \%$ acetone, Epon 812 resin infiltration. HeLa cells with mitochondrially expressed protein. f) mEos4a; survival is very poor, with a single weakly fluorescent cell found in the dish. g) "Eos5", green channel; survival is excellent, with all cells in the dish highly fluorescent. h) "Eos5", photoconverted and shown in red channel. Panels i-k: CLEM of "Eos5" in Epon 812 resin. A HeLa cell with mitochondrially expressed "Eos5". i) Confocal fluorescence image. j) TEM image. k) CLEM image.

References

[1] Paez-Segala et al., Nature Methods 12: 215-218 (2015).

[2] Kopek et al., Nature Protocols 12: 916-946 (2017).

[3] Menon et al., Wiley Interdisciplinary Reviews in Developmental Biology 2: 647-670 (2013).

[4] Jia et al., Journal of Neurobiology 24: 1025-1044 (1993). 